\title{
Copper, silver, and titania nanoparticles do not release ions under anoxic conditions and release only minute ion levels under oxic conditions in water: Evidence for the low toxicity of nanoparticles
}

\author{
Marina R. Mulenos ${ }^{1} \cdot$ Jiaqi Liu ${ }^{2} \cdot$ Henry Lujan $^{1} \cdot$ Binglin Guo $^{2} \cdot$ Eric Lichtfouse $^{3}$ D $\cdot$ Virender K. Sharma $^{2}$. \\ Christie M. Sayes ${ }^{1}$
}

\begin{abstract}
It is widely assumed that the discharge of nanoparticles into the environment may cause adverse effects on organisms. Nonetheless, so far most nanoparticles have demonstrated little to no observed hazards in multiple biological test systems. It is not until nanoparticles undergo transformations, e.g., release of metal ions, that most environmental toxicities are induced, yet the ionization of nanoparticles in natural oxic and anoxic conditions is poorly known. We hypothesized that in anaerobic conditions, where oxidation is absent or limited, metal nanoparticles should not release metal ions. We investigated the transformation of three commercially produced materials, i.e., copper nanoparticles, silver nanoparticles, and titania nanocrystals with an average particle size of $50 \mathrm{~nm}$. The nanoparticles were subjected to different environmental conditions including oxic/anoxic suspension, incubation with natural organic matter, and $\mathrm{pH}$ gradient, then subsequently analyzed for zeta potential, hydrodynamic diameter, concentration of released metal ions, and generation of reactive oxygen species. Transmission electron microscopy was used to assess morphological changes. Under oxic conditions, results show that only $9.4 \mu \mathrm{g} / \mathrm{mL}$ of copper ions and $6.9 \mu \mathrm{g} / \mathrm{mL}$ of silver ions were released from nanoparticles, when continuously stirred over $48 \mathrm{~h}$. These levels are low compared to levels found in natural media. Moreover, under anoxic conditions, an insignificant amount of copper ions of $0.9 \mu \mathrm{g} / \mathrm{mL}$ and silver ions, of $0.2 \mu \mathrm{g} / \mathrm{mL}$, were released. For titania nanoparticle, less than $0.05 \mu \mathrm{g} /$ $\mathrm{mL}$ ions were released under either oxic or anoxic conditions. Overall, our findings reveal the absence of ion release under anoxic conditions, and the very low levels of ion released under oxic conditions. As a consequence, the toxicity of $\mathrm{Cu}, \mathrm{Ag}$, and Ti nanoparticles should be very low in natural media.
\end{abstract}

Keywords Anoxic conditions $\cdot$ Copper nanoparticles $\cdot$ Silver nanoparticles $\cdot$ Titania nanocrystals $\cdot$ Natural organic matter $\cdot$ Reactive oxygen species $\cdot$ Dissolved metal ions

Christie M. Sayes

Christie_Sayes@baylor.edu

1 Department of Environmental Science, Baylor University, One Bear Place \#97266, Waco, TX 76798-7266, USA

2 Program for the Environment and Sustainability, Department of Environmental and Occupational Health, School of Public Health, Texas A\&M University, College Station, TX, USA

3 CNRS, IRD INRAE, Coll France, CEREGE, Aix-Marseille University, 13100 Aix-en-Provence, France

\section{Introduction}

Engineered nanomaterials represent a wide range of advanced materials intentionally added to industrial and consumer products and processes in an effort to increase their performance (Pati et al. 2016; Ahn et al. 2018; Alivio et al. 2018; Kim et al. 2018; Westerhoff et al. 2018; Vishnuvarthanan and Rajeswari 2019). Nanoparticles range from insulating to semiconductive to conductive elemental compositions. Manufactures generate nano-enabled products by embedding nanoparticles into a matrix or coating the surface of a product (Keller and Lazareva 2014; Bäuerlein et al. 2017; Kaur et al. 2018). However, when nanomaterials emulsify in a gelatinous substance or suspend in a liquid, they tend to migrate from the mixture into the surrounding environment (Westesen et al. 1997). These embedded 
nanoparticles have the potential to release from products and transform under environmental conditions (Adegboyega et al. 2014; Garner and Keller 2014; Amde et al. 2017; Joo and Zhao 2017; Ortelli et al. 2017; Zhang et al. 2018; Simeone et al. 2019).

Metal nanoparticles are typically produced in their zerovalent forms, indicating that the outer shell of the particle has a valence value of zero. Examples of zerovalent nanoparticles are copper and silver nanoparticles (Akaighe et al. 2011; Gao et al. 2019; Hedberg et al. 2019). Similarly, metal oxide nanoparticles, e.g., titanium dioxide nanocrystals, are stable and remain intact when stored as dry powders (Sharma 2009; Li et al. 2019). Metal and metal oxide nanoparticles suspended in aqueous solutions may transform when exposed to natural organic matter, oxic/anoxic conditions, and ultraviolet light (He et al. 2011; Lowry et al. 2012; Yin et al. 2012; Sharma et al. 2015; Wang et al. 2017; Yin et al. 2017a, b; Pham et al. 2018; Hedberg et al. 2019). During this reaction nanoparticles may transform from their pristine engineered zerovalent state to surface-charged, ion-dissociated, agglomerated/ aggregated forms (Hou et al. 2013; Sharma and Zboril 2017). These transformation processes may have negative effects on human health and ecological systems due to the possible release of toxic metal ions and reactive oxygen species (Georgantzopoulou et al. 2018; Rai et al. 2018; Wang et al. 2018; $\mathrm{Li}$ et al. 2019). The focus of the present paper is to understand the transformation of selected nanoparticles under different environmental conditions.

The studied nanoparticles are copper nanoparticles, silver nanoparticles, and titania nanocrystals, which have been applied in thousands of consumer products (Garner et al. 2017; Sharma et al. 2019). The physicochemical characteristics of the nanoparticles under aqueous environment were studied with the following aims: (i) dissolution under stirring oxic and anoxic conditions to evaluate the release of toxic metal ions, (ii) quantification of reactive oxygen species release under both oxic and anoxic conditions, (iii) the solution was exposed to natural organic matter and imaged utilizing transmission electron microscopy which was determined to monitor the stability of the nanoparticles, and (iv) the $\mathrm{pH}$ of the aqueous solution was varied from acidic to alkaline $\mathrm{pH}$, and measurements of zeta potential and hydrodynamic diameters were determined to learn the change in surface charge and size. The results presented herein are expected to aid in the assessments of hazards, exposures, and risks of engineered nanomaterials that transform after entering to the aquatic environment.

\section{Experimental}

\section{Experimental design}

Copper and silver nanoparticles were purchased from Sigma-Aldrich at the highest percent purity offered (St. Louis, Missouri, USA; copper nanopowder, 40 to $60 \mathrm{~nm}$ particle size, greater than or equal to $99.5 \%$ trace metals basis; silver nanopowder, lower than $100 \mathrm{~nm}$ particle size, 99.5\% trace metals basis). Titania nanocrystals were purchased from Evonik (Essen, Germany; AEROXIDE ${ }^{\circledR} \mathrm{P} 25$; lower than $100 \mathrm{~nm}$ particle size; $97 \%$ trace metal basis). Density reported by the manufacturers was 8.0, 10.5, and $4.2 \mathrm{~g} / \mathrm{cm}^{3}$ for copper nanoparticles, silver nanoparticles, and titania nanocrystals, respectively.

The experimental design, as shown in Fig. 3, incorporates four independent studies designed to characterize surface charge and size through zeta potential and hydrodynamic size measurements, morphological information from transmission electron microscopy, metal impurities, and released ion concentration using inductively coupled plasma mass spectrometry, and generation of reactive oxygen by ultraviolet spectroscopy. For all experiments, an incubation or exposure time period of $48 \mathrm{~h}$ at room temperature, i.e., $2{ }^{\circ} \mathrm{C}$, was used. 48 -h incubation time was used due to toxicological studies being 24- and/or 48-h long experiments (Lewinski et al. 2008; Selvaraj et al. 2014). The particle concentration was $100 \mu \mathrm{g} / \mathrm{mL}$ in $18.2 \mathrm{~m} \Omega$ Milli-Q ultrapure water, unless otherwise noted. All studies were performed in triplicate.

\section{Nanomaterial aqueous suspension stirring under oxic and anoxic conditions}

Aqueous suspensions of nanomaterials were agitated via magnetic stir bar from Thermo Fischer Scientific (Waltham, Massachusetts, USA) in either oxygen-saturated water or nitrogen-purged water. Oxygen-saturated and nitrogen-purged solutions were produced by purging pressurized air and nitrogen gas into a parafilm-covered beaker throughout the agitation, respectively (Zhang et al. 2018). Every $6 \mathrm{~h}$, a 10-mL aliquot was taken from the top of each suspension and underwent centrifugal ultrafiltration before preparation for analyses via digestion with a nitric and hydrochloric acid mixture, $67 \%$ and $36 \%$, respectively, at a 3:1 ratio (De Jong et al. 2008). Briefly, a particle concentrating centrifugal cartridge was used (Amicon Ultra 15, Millipore, Burlington, Massachusetts, USA). The $10-\mathrm{mL}$ aliquot was added to each cartridge and centrifuged for $15 \mathrm{~min}$ at $5000 \mathrm{rpm}$. After each procedure, approximately $500 \mu \mathrm{L}$ of concentrated suspension 
remained in the receptacle. Both the filtrate and the concentrated sample was digested and analyzed for total metal content.

Inductively coupled plasma mass spectrometry Agilent 7900 (Santa Clara, California, USA) instrument was used to measure metal ion concentrations from the dissolution of studied nanoparticles. Briefly, the sample digests were placed in the autosampler and ran in triplicate along with an environmental internal standard which contained lithium, scandium, germanium, rhodium, indium, terbium, lutetium, and bismuth from Agilent (Santa Clara, California, USA). It is noted that titanium ions are difficult to analyze using inductively coupled plasma mass spectrometry. However, in an effort to maintain consistency among nanoparticle analyses, we subjected titania to the same sample preparation process and mass spectrometry analyses as copper and silver nanoparticle samples. The concentrations of metal ions were calculated through a linear regression analysis against prepared standards from Agilent (Santa Clara, California, USA). Method blanks and acid blanks were measured as well. Data analysis was performed with Excel (Microsoft, Redmond, Washington, USA.) After data analysis, mass balance calculations verified total amount of released metal ions.

\section{Nanomaterial exposure to ultraviolet radiation and generation of reactive oxygen species}

Each nanoparticle suspension was vortexed utilizing a Vortex-Genie 2 (Scientific Industries; Bohemia, New York, USA) for $30 \mathrm{~s}$, and then placed under shortwave ultraviolet lamp at $254 \mathrm{~nm}$ (Fisher Scientific, Hampton, New Hampshire, USA) for 15 min, and immediately analyzed for reaction oxygen species generation. 2',7'-dichlorofluorescein diacetate (Cell Biolabs, Inc.; San Diego, California, USA) was used to measure the concentration of reactive oxygen species, described elsewhere (Romoser et al. 2011). Briefly, a $100 \mu \mathrm{M}$ of the diacetate solution was added to $1-\mathrm{mL}$ aliquot of particle suspensions and incubated at $37^{\circ} \mathrm{C}$ for $15 \mathrm{~min}$. 1000 $\mu \mathrm{M}$ hydrogen peroxide (Fisher Scientific, Hampton, New Hampshire, USA) was used as a positive control for determining reactive oxygen species concentration. Ultrapure water served as a negative control. Fluorescence of the sample was measured at an excitation of $480 \mathrm{~nm}$ and an emission of $530 \mathrm{~nm}$ using a Synergy Mx Multi-Mode Microplate Reader (BioTek Instruments, Inc.; Winooski, Vermont, USA). Analyses involved the detection and the measurement of general reactive oxygen species, i.e., super oxide anion, hydroxyl radical, and singlet oxygen.

\section{Nanomaterial incubation with natural organic matter and analyses by transmission electron microscopy}

Nanoparticle suspensions were subjected to an addition of mass equivalent concentration of Suwannee River natural organic matter (International Humic Substances Society; Denver, Colorado, USA). Suwannee River natural organic matter is used as the universal standard of natural organic matter. First, the organic matter was suspended in ultrapure water at a concentration of $100 \mu \mathrm{g} / \mathrm{mL}$ (Liu and Hurt 2010). After $30 \mathrm{~s}$ agitation on a Vortex-Genie 2 (Scientific Industries; Bohemia, New York, USA), each nanoparticle suspension was added at equal parts volume and vortexed for an additional $30 \mathrm{~s}$. Samples were left to incubate at room temperature; subsequent vortex occurs for 30-s interval every $8 \mathrm{~h}$ for $48 \mathrm{~h}$ in total.

Transmission electron microscopy was performed for nanoparticles and nanoparticle-organic matter mixtures (Stebounova et al. 2011). Details of sample preparation include a $10-\mu \mathrm{L}$ droplet of nanoparticle suspension which was set on parafilm, and a formvar-coated copper grid from Electron Microscopy Sciences (Hatfield, Pennsylvania, USA) was placed over top for $5 \mathrm{~min}$. The grid was then removed and dried before imaging. The samples were imaged with a JEOL 2010 microscope (JEOL Ltd.; Tokyo, Japan), operating at $60 \mathrm{kV}$. Post-image analyses were performed with ImageJ software.

\section{Change in nanomaterial $\mathrm{pH}$ and analysis via dynamic light scattering}

A range of nanomaterial suspensions, inclusive of 0.1 to $1000 \mu \mathrm{g} / \mathrm{mL}$, were titrated using the Malvern MPT-2 Autotitrator in parallel with the Zetasizer Nano-ZS (Malvern Panalytical Ltd.; Malvern, United Kingdom). This combination allowed titration over a wide $\mathrm{pH}$ range and thus made it possible to determine the isoelectric point. Particles were automatically titrated from $\mathrm{pH} 12.0$ to 2.0 by adding $0.25 \mathrm{M}$ hydrochloric acid and $0.25 \mathrm{M}$ sodium hydroxide (Fisher Scientific, Hampton, New Hampshire, USA). A folded capillary cell with electrodes (Malvern Panalytical Ltd.; Malvern, United Kingdom) was used for all measurements where $500 \mu \mathrm{L}$ of nanoparticle suspension was loaded. At every $0.2 \mathrm{pH}$ unit, the zeta potential and hydrodynamic size were determined. These experiments collected eleven measurements, and the experiment was repeated in triplicate. The isoelectric point was determined using the Malvern Software Version 5.03. 


\section{Statistical analyses}

Statistical analyses were performed using SAS 9.4 software (SAS Institute Inc.; Cary, North Carolina, USA). Each experimental value was compared to the corresponding control value for each experiment. One-way ANOVA test was performed at each sampling time. When the F-test from ANOVA shows significance, the Dunnett's or Dunn's test was used to compare means from the control group. Statistical significance versus control group was established at levels corresponding to $p$ values lower than 0.05 . Experiments were conducted with $\mathrm{n}=3$ and in triplicate.
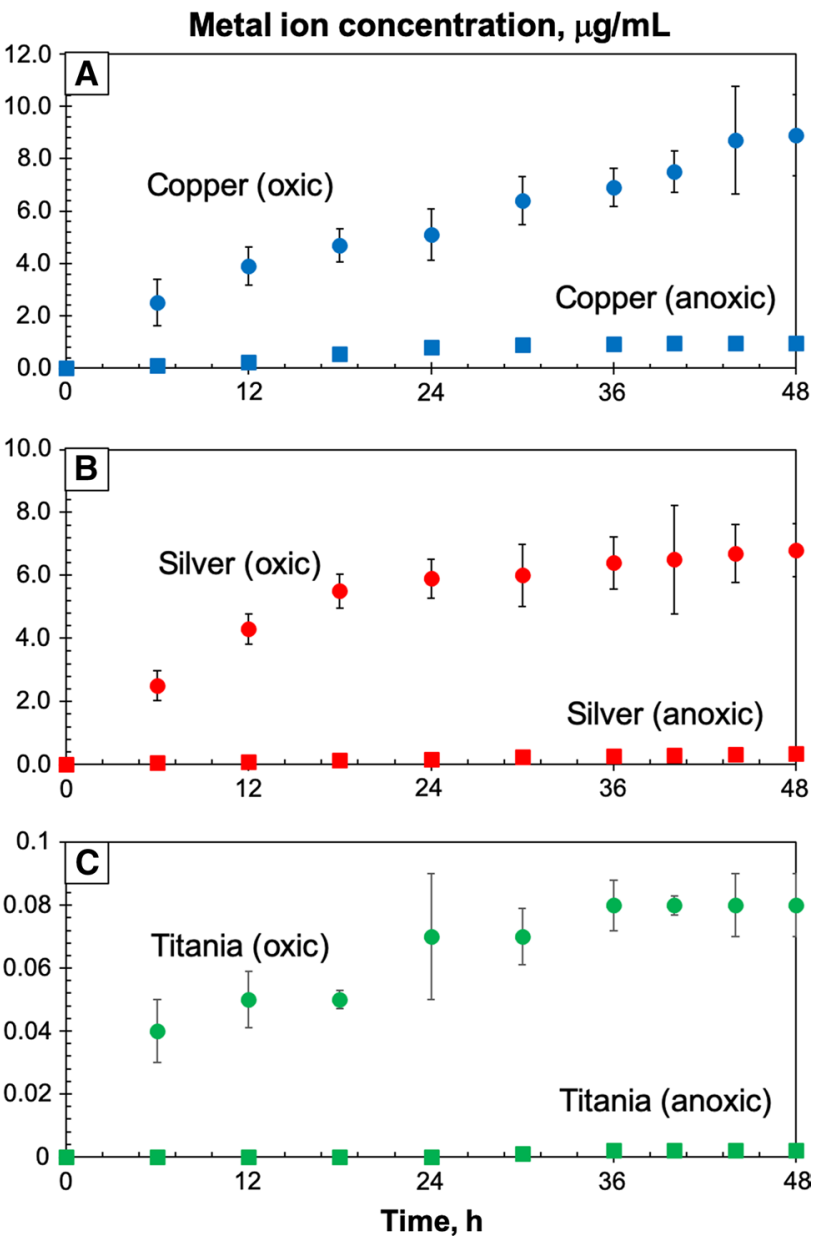

Fig. 1 Release of metal ions from nanoparticles is dependent on oxic conditions. Metal ion concentrations under air-saturated (oxic) or nitrogen-purge (anoxic) for a copper, $\mathbf{b}$ silver, and $\mathbf{c}$ titania particle suspensions in water. Solid lines represent data collected from air-saturated system, while dotted lines represent data collected from nitrogen-purge system. Each data set represents the average and the standard deviation $(n=9)$

\section{Results and discussion}

\section{Dissolution under oxic and anoxic condition}

Two orthogonal approaches, i.e., oxic and anoxic conditions, were used to measure the ion release of each investigated nanomaterial. Figure 1 shows the concentrations of released metal ions over time under both conditions. Copper and silver nanoparticles released increasing amounts, $9.4 \mu \mathrm{g} / \mathrm{mL}$ and $6.9 \mu \mathrm{g} / \mathrm{mL}$, respectively, of dissolved ions with time from the particle surfaces when stirred and aerated. Silver nanoparticles dissolved at the faster rate between $0-20 \mathrm{~h}$ than the rate observed between $20-48 \mathrm{~h}$ (Fig. 1b). It is possible that an oxide shell formed on the surface of the silver nanoparticles during the dissolution in the initial phase, which would decrease the ion release rate (Akaighe et al. 2011). As expected, titania nanocrystals did not produce a significant amount, lower than $0.05 \mu \mathrm{g} /$ $\mathrm{mL}$, of ions under the oxic condition (Fig. 1c).

Under the anoxic conditions, i.e., stirred and purged with nitrogen gas, a significantly lesser amount of dissolved ions were released from the nanomaterials (Fig. 1). Copper nanoparticles released $0.9 \mu \mathrm{g} / \mathrm{mL}$, silver nanoparticles released $0.2 \mu \mathrm{g} / \mathrm{mL}$, and no measurable ions were released from titania nanocrystals. This indicates that dissolved oxygen in solution plays a pivotal role in releasing dissolved metal ions from nanomaterials. Previous investigations in the case of silver nanoparticles also showed similar observations, e.g., $\mathrm{Ag}^{0}+\mathrm{O}_{2} \rightarrow \mathrm{Ag}^{+}+\mathrm{O}_{2}^{-}$(He et al. 2011, 2012; Rong et al. 2019).

\section{Generation of reactive oxygen species under UV irradiation}

Figure 2 shows the generation of reactive oxygen species from the surfaces of each nanomaterial used in the study after 15 min irradiation with ultraviolet light. In general, reactive oxygen species increases as particle concentration increases. However, the increase in reactive species production differs among the three different metal particle types. Copper nanoparticles follow a dose-response relationship, i.e., as much as $1250 \pm 8 \%$ relative fluorescence units at $1000 \mu \mathrm{g} / \mathrm{mL}$ to as little as $800 \pm 10 \%$ relative fluorescence units at $0.1 \mu \mathrm{g} / \mathrm{mL}$, across all concentrations, and the sample produced the most reactive oxygen species of all samples when suspended in water. The term "relative fluorescence units" is a unit of measurement used in the fluorescence detection analysis and, for this experiment, is directly proportional to the concentration of reactive oxygen species in the sample (Nasseri et al. 2018). Silver nanoparticles did not follow a dose-response relationship, 

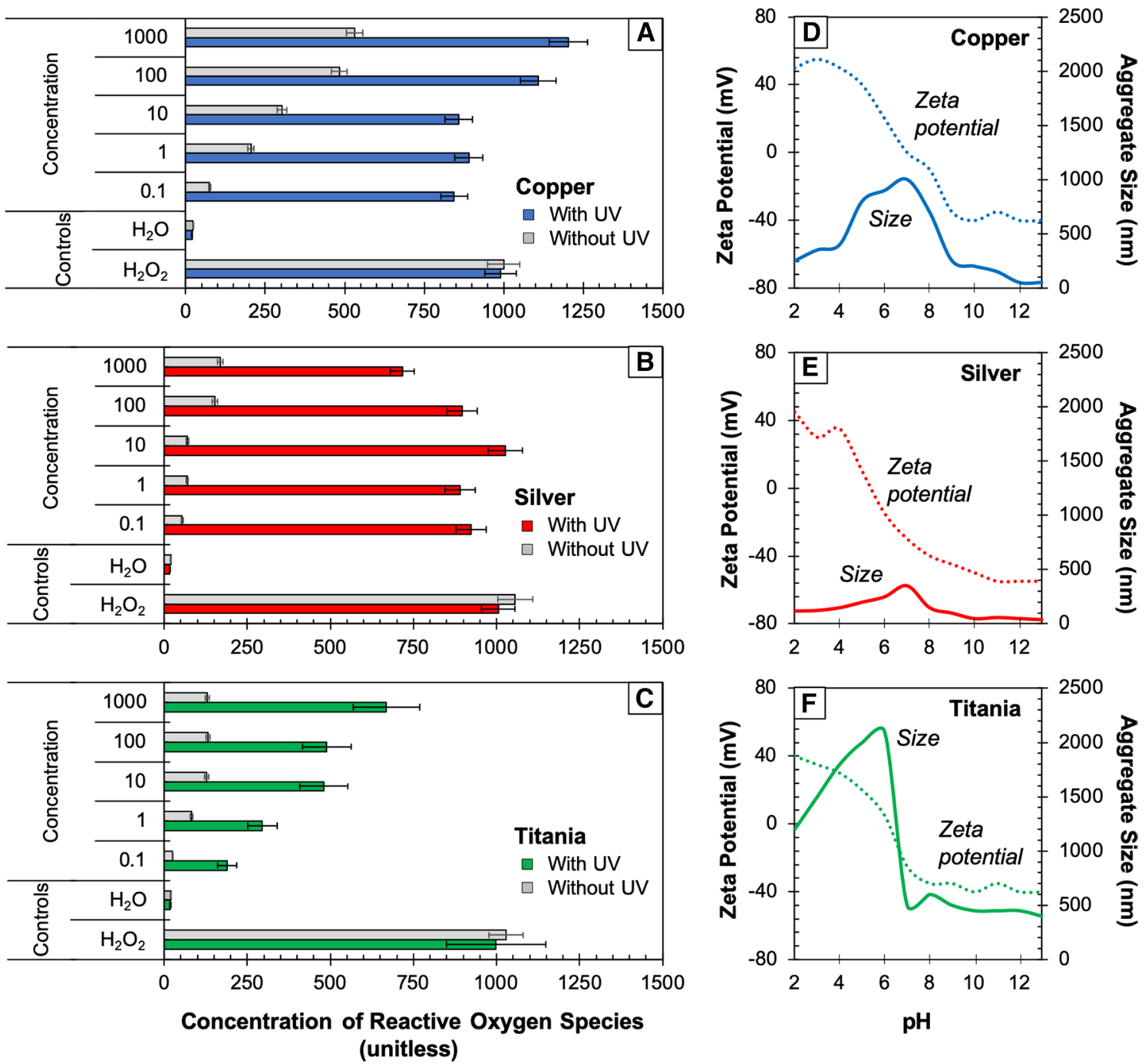

Fig. 2 Reactive oxygen species concentration is dependent on nanoparticle concentration. a Copper nanoparticles follow a concentration-dependent relationship across all particle concentrations. b Silver nanoparticles follow a concentration-dependent relationship from $0.1-10 \mathrm{ug} / \mathrm{mL}$, but the reactive oxygen species concentration decreases from $10-1000 \mathrm{ug} / \mathrm{mL}$. c Titania nanoparticles produce the

e.g., the highest concentration of species detected was $1050 \pm 8 \%$ relative fluorescence units at $10 \mu \mathrm{g} / \mathrm{mL}$ and the lowest concentration of species detected was $725 \pm 9 \%$ relative fluorescence units at $1000 \mu \mathrm{g} / \mathrm{mL}$. The literature has shown that silver nanoparticles have the ability to generate reactive oxygen species with and without ultraviolet irradiation. The presence of oxygen in aerated solution upon irradiation tends to produce reactive oxygen species, e.g., $\mathrm{O}_{2}^{-\bullet}$ (Adegboyega et al. 2016; Ranjan and Ramalingam 2016; Rong et al. 2019). However, the produced reactive oxygen species may interact with released metal ions to reproduce silver nanoparticles in a feedback mechanism (He et al. 2011). least amount of reactive oxygen species and the concentrations at statistically insignificant across all particle concentrations. All particle samples were exposed to ambient light for $1 \mathrm{~h}$ before analyses. Each data set represents the average and the standard deviation $(n=9)$. Isoelectric point and hydrodynamic diameter measurements of $\mathbf{d}$ copper, e silver, and $\mathbf{f}$ titania nanoparticles in water over $\mathrm{pH}$

Lastly, titania nanocrystals produced the least amount of reactive oxygen species over a dose-response relationship, e.g., as much as $675 \pm 18 \%$ relative fluorescence units at $1000 \mu \mathrm{g} / \mathrm{mL}$ to as little as $210 \pm 22 \%$ relative fluorescence units at $0.1 \mu \mathrm{g} / \mathrm{mL}$. Taken together, these data suggest that the cumulative effect of production and consumption of oxygen would ultimately determine the concentration of reactive oxygen species upon irradiation of nanomaterials in water (Nasseri et al. 2018; Irandost et al. 2019; Li et al. 2019). 


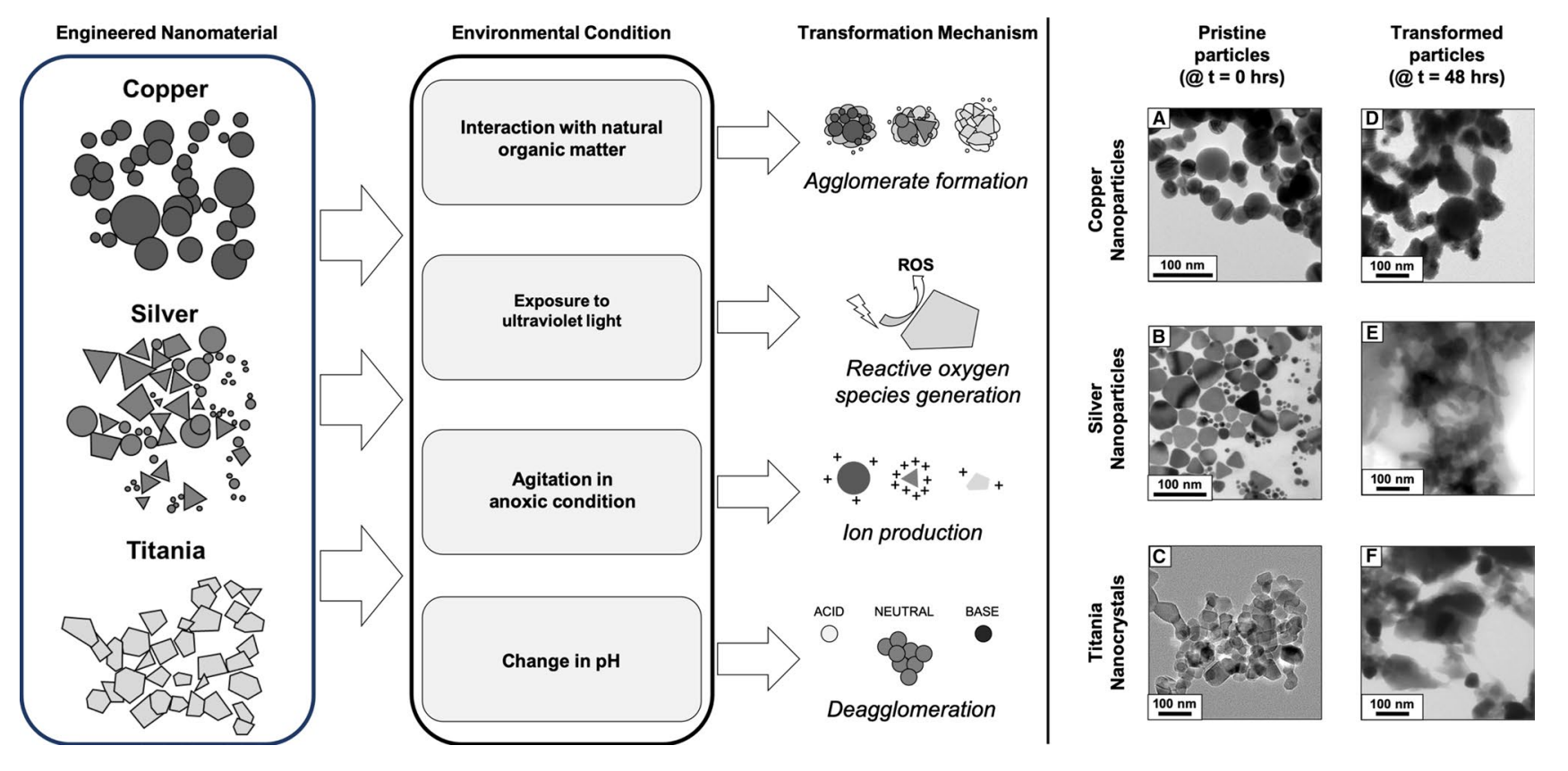

Fig. 3 (Left) Experimental design used to observe and measure nanomaterial transformations. The conditions of each experiment are as follows: interaction with natural organic matter (room temperature, ambient light, ambient oxygen, and neutral $\mathrm{pH}$ ); exposure to ultraviolet light (no natural organic matter, room temperature, ambient oxygen, and neutral $\mathrm{pH}$ ); agitation with dissolved oxygen (no natural organic matter, room temperature, ambient light, and neutral $\mathrm{pH}$ ); and

\section{Effect of natural organic matter}

Figure 3 shows the transmission electron micrographs of the three particle systems and their morphological features in the absence and presence of natural organic matter in water. In water, shapes of the particles are generally spherical, triangular, and trapezoidal for copper nanoparticles, silver nanoparticles, and titania nanocrystals, respectively. In the presence of organic matter, all particles underwent dissolution to the extent where each individual particle could no longer be distinguished. The moieties of natural organic matter interact with the surface of the particle. The interaction with multiple particles may increase the overall size of the nanoparticle or possibly induce aggregation. These interactions can be physical or chemical or both (Sani-Kast et al. 2017; Huang et al. 2019). For example, silver ions, likely produced from dissolution of silver nanoparticles under oxidic conditions, would interact with sulfur-containing groups of organic matter through complexation (Ubaid et al. 2019). The transformation of silver nanoparticles seems to be the highest in the presence of natural organic matter compared to either the original sample or the absence of any organic species, where many particles agglomerated and morphed into a large agglomerated structure. In the natural environment, silver sulfide nanoparticles, thought change in $\mathrm{pH}$ (no natural organic matter, room temperature, ambient light, and ambient oxygen). (Right) Transmission electron microscopy image of a copper, $\mathbf{b}$ silver, and $\mathbf{c}$ titania particles taken immediately after suspension in ultrapure water. Transmission electron micrographs of $\mathbf{d}$ copper, e silver, and $\mathbf{f}$ titania particles taken $48 \mathrm{~h}$ after incubation with natural organic matter in water

to be reproduced from dissociated silver nanoparticles in a feedback mechanism, have been found (Sharma et al. 2015; Ubaid et al. 2019). Similarly, copper sulfide has also been seen in the aquatic environment (Sharma et al. 2015). Other moieties of organic matter, such as phenols and hydroquinone, may also be involved in the complexation processes. Titania nanocrystals do not dissolve into their respective metal ions, and therefore, would only interact with organic species through physical phenomena, i.e., adsorption.

\section{Effect of $\mathrm{pH}$}

Figure 2 shows the variation of zeta potentials and hydrodynamic diameters with $\mathrm{pH}$ in the range from 2.0 to 13.0. All three samples followed the same trend; at a low $\mathrm{pH}$, the zeta potentials have positive values, while at a high $\mathrm{pH}$, they possess negative values. The measurements of zeta potentials of the three nanomaterials over a wide $\mathrm{pH}$ were used to deduce the isoelectric points, a.k.a. conductometric titrations, which is the $\mathrm{pH}$ of the nanomaterial suspension where zeta potential is neutral. The isoelectric points are 7.0, 6.5, and 5.5 of copper nanoparticles, titania nanocrystals, and silver nanoparticles, respectively. These values suggest that the studied nanomaterials would be mostly negatively charged at environmentally pH conditions (Pham et al. 2018). 
Hydrodynamic diameters of the three materials are also shown in Fig. 2. Titania had the largest particle size range from 200 to $2200 \mathrm{~nm}$, then copper with a range of 100-1000 nm, and then silver, which did not change much, at 100 to $200 \mathrm{~nm}$. All three samples followed similar trends with a small hydrodynamic diameter at both highly acidic and alkaline $\mathrm{pH}$. The maximum sizes are at their isoelectric points.

\section{Conclusion}

The physicochemical measurements, shown in Figs. 1, 2, 3 and 4, demonstrate the role of different environmental parameters on the transformation of the nanomaterials. The nanoparticles in natural waters are not static and their physicochemical properties would change dynamically over time and spatial coordinates. The influence of these conditions on the interactions of the nanoparticles is shown in Fig. 4. The investigated nanoparticles of negatively charged surfaces under environmental $\mathrm{pH}$, based on isoelectric point measurements, would interact with different moieties of organic matter. These physical and chemical interactions likely cause aggregation of the nanoparticles (Shevlin et al. 2018). These interactions depend on the type of metal ions. For example, titania nanocrystals do not release ions and only exhibit physical interactions; copper nanoparticles readily release ions and have both physical and chemical interactions; and silver nanoparticles also readily release ions and due to complexation processes produced the most significant physical and chemical interactions. The interaction of organic species may stabilize nanomaterials for months. In consequence, this phenomenon may promote nanomaterial intercalation within microorganisms, accumulation within aquatic organisms, or

\section{Ultraviolet Light Irradiation}

\section{Reactive Oxygen Species Generation}

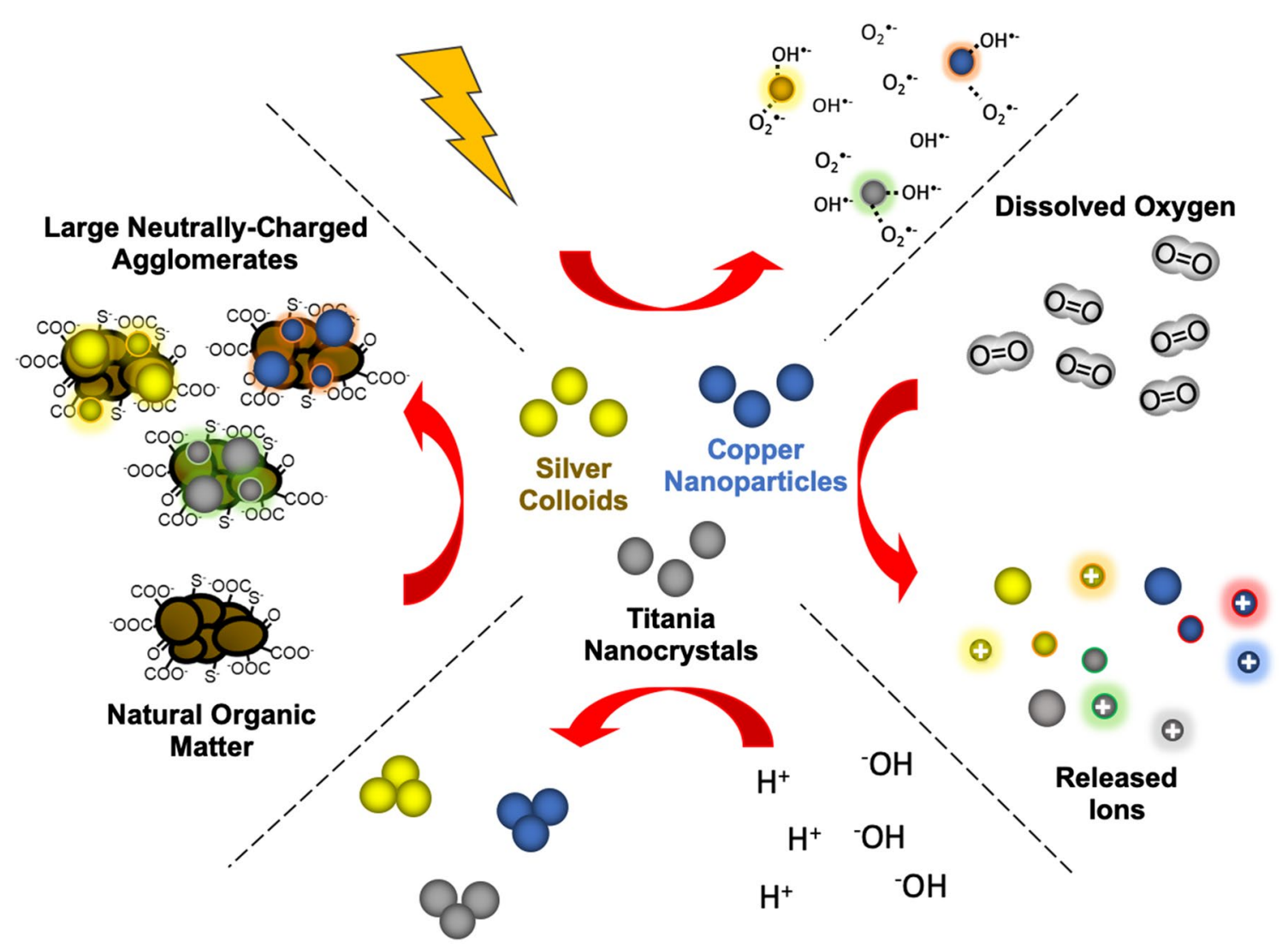

Small Surfaced-Charged Aggregates

Fig. 4 Potential mechanisms of nanoparticle transformations in environmental conditions. When irradiated with ultraviolet light, particles generate reactive oxygen species. In oxic conditions, particles release ions into surrounding matrix. In strongly acidic or basic conditions, particles are less likely to severely aggregate. In the presence of natural organic matter, particles seem to stabilize with after adsorption with organic matter. Taken together, these transformations forecast potential environmental effects 
transportation through water (Quigg et al. 2013). This is important for assessing the safety of these materials because such organisms are exposed to these materials after their release into the environment.

In addition to complexation with natural organic matter, nanomaterials are exposed to ultraviolet radiation from the sun. In the natural water, organic matter can also absorb radiation and would generate reactive oxygen species, such as ${ }^{1} \mathrm{O}_{2},{ }^{\bullet} \mathrm{OH}$, and $\mathrm{O}_{2}^{\bullet-}$ (Zhang et al. 2014, 2017). The concentrations of reactive species depend on nature and sources organic species as well as the matrices containing metal ions (Sharma et al. 2019). Therefore, the release of metal ions from the nanomaterials is important. Titania nanocrystals did not react differently in either oxic or anoxic conditions. This is in contrast to copper and silver nanoparticles, which had nine times and seven times increase in metal ion concentration, respectively, over time. Both copper and silver nanoparticles readily produce reactive oxygen species after ultraviolet radiation, while titania nanocrystals do not. Similarly, copper and silver nanoparticles readily release ions from their particle surfaces, while titania nanocrystals do not. These relationships are correlative and possibly causative. It has been seen that natural light induces dissociation of ions from nanoparticle surfaces. Furthermore, surface oxidation on metal nanomaterial surfaces is inversely related to reactive oxygen species and directly proportional to metal ion generation (Albanese et al. 2012; Fu et al. 2014). Additionally, the size of the nanoparticle may also influence reactive oxygen species and metal ion generation (Carlson et al. 2008).

The results of the study will aid in characterizing the transportation and transformation of nanomaterial predictions as well as their possible fate and effects in the aquatic environment. The transformation of metal-based nanomaterials in the environment is a culmination of $\mathrm{pH}$, dissolved oxygen content, natural organic matter concentration, and light illumination. The production of reactive oxygen species in environmental systems may forecast potential toxicological effects. This research will enable the understanding of the chemical and toxicological mechanisms of metal particles and the resulting transformed products in the aquatic environment.

\section{Compliance with ethical standards}

Conflict of interest The authors declare no conflict of interest

\section{References}

Adegboyega NF, Sharma VK, Siskova KM, Vecerova R, Kolar M, Zbořil R, Gardea-Torresdey JL (2014) Enhanced formation of silver nanoparticles in $\mathrm{Ag}+$-NOM-iron (II, III) systems and antibacterial activity studies. Environ Sci Technol 48(6):3228-3235

Adegboyega NF, Sharma VK, Cizmas L, Sayes CM (2016) UV light induces Ag nanoparticle formation: roles of natural organic matter, iron, and oxygen. Environ Chem Lett 14(3):353-357

Ahn EC, Wong HSP, Pop E (2018) Carbon nanomaterials for nonvolatile memories. Nat Rev Mater 3(3):1-15

Akaighe N, MacCuspie RI, Navarro DA, Aga DS, Banerjee S, Sohn M, Sharma VK (2011) Humic acid-induced silver nanoparticle formation under environmentally relevant conditions. Environ Sci Technol 45(9):3895-3901

Albanese A, Tang PS, Chan WCW (2012) The effect of nanoparticle size, shape, and surface chemistry on biological systems. Annu Rev Biomed Eng 14:1-16

Alivio TEG, Fleer NA, Singh J, Nadadur G, Feng M, Banerjee S, Sharma VK (2018) Stabilization of Ag-Au bimetallic nanocrystals in aquatic environments mediated by dissolved organic matter: a mechanistic perspective. Environ Sci Technol 52(13):7269-7278

Amde M, Liu J-F, Tan Z-Q, Bekana D (2017) Transformation and bioavailability of metal oxide nanoparticles in aquatic and terrestrial environments. A review. Environ Pollut 230:250-267

Bäuerlein PS, Emke E, Tromp P, Hofman JAMH, Carboni A, Schooneman F, de Voogt P, van Wezel AP (2017) Is there evidence for man-made nanoparticles in the Dutch environment? Sci Total Environ 576:273-283

Carlson C, Hussain SM, Schrand AM, Braydich-Stolle LK, Hess KL, Jones RL, Schlager JJ (2008) Unique cellular interaction of silver nanoparticles: size-dependent generation of reactive oxygen species. J Phys Chem B 112(43):13608-13619

Fu PP, Xia Q, Hwang H-M, Ray PC, Yu H (2014) Mechanisms of nanotoxicity: generation of reactive oxygen species. J food Drug Anal 22(1):64-75

Gao X, Rodrigues SM, Spielman-Sun E, Lopes S, Rodrigues S, Zhang Y, Avellan A, Duarte RMBO, Duarte A, Casman EA (2019) Effect of soil organic matter, soil $\mathrm{pH}$, and moisture content on solubility and dissolution rate of CuO NPs in soil. Environ Sci Technol 53(9):4959-4967

Garner KL, Keller AA (2014) Emerging patterns for engineered nanomaterials in the environment: a review of fate and toxicity studies. J Nanopart Res 16(8):2503

Garner KL, Suh S, Keller AA (2017) Assessing the Risk of Engineered Nanomaterials in the Environment: development and Application of the nanoFate Model. Environ Sci Technol 51(10):5541-5551

Georgantzopoulou A, Almeida Carvalho P, Vogelsang C, Tilahun M, Ndungu K, Booth AM, Thomas KV, Macken A (2018) Ecotoxicological effects of transformed silver and titanium dioxide nanoparticles in the effluent from a lab-scale wastewater treatment system. Environ Sci Technol 52(16):9431-9441

He D, Garg S, Waite TD (2012) H2O2-mediated oxidation of zerovalent silver and resultant interactions among silver nanoparticles, silver ions, and reactive oxygen species. Langmuir 28(27):10266-10275

He D, Jones AM, Garg S, Pham AN, Waite TD (2011) Silver nanoparticle - reactive oxygen species interactions: application of a charging - discharging model. J Phys Chem C 115(13):5461-5468

Hedberg J, Blomberg E, Odnevall Wallinder I (2019) In the search for nanospecific effects of dissolution of metallic nanoparticles at freshwater-like conditions: a critical review. Environ Sci Technol 53(8):4030-4044

Hou W-C, Stuart B, Howes R, Zepp RG (2013) Sunlight-driven reduction of silver ions by natural organic matter: formation and transformation of silver nanoparticles. Environ Sci Technol 47(14):7713-7721

Huang Y-N, Qian T-T, Dang F, Yin Y-G, Li M, Zhou D-M (2019) Significant contribution of metastable particulate organic matter 
to natural formation of silver nanoparticles in soils. Nat Commun 10(1):1-8

Irandost M, Akbarzadeh R, Pirsaheb M, Asadi A, Mohammadi P, Sillanpää M (2019) Fabrication of highly visible active N, S co-doped TiO2@MoS2 heterojunction with synergistic effect for photocatalytic degradation of diclofenac: mechanisms, modeling and degradation pathway. J Mol Liq 291:111342

Joo SH, Zhao D (2017) Environmental dynamics of metal oxide nanoparticles in heterogeneous systems: a review. J Hazard Mater 322:29-47

Kaur M, Kaur M, Sharma VK (2018) Nitrogen-doped graphene and graphene quantum dots: a review onsynthesis and applications in energy, sensors and environment. Adv Coll Interface Sci 259:44-64

Keller AA, Lazareva A (2014) Predicted releases of engineered nanomaterials: from global to regional to local. Environ SciTechnol Lett 1(1):65-70

Kim Y, Smith JG, Jain PK (2018) Harvesting multiple electron-hole pairs generated through plasmonic excitation of Au nanoparticles. Nat Chem 10(7):763-769

Lewinski N, Colvin V, Drezek R (2008) Cytotoxicity of nanoparticles. Small 4(1):26-49

Li K, Qian J, Wang P, Wang C, Fan X, Lu B, Tian X, Jin W, He X, Guo W (2019) Toxicity of three crystalline TiO2 nanoparticles in activated sludge: bacterial cell death modes differentially weaken sludge dewaterability. Environ Sci Technol 53(8):4542-4555

Liu J, Hurt RH (2010) Ion release kinetics and particle persistence in aqueous nano-silver colloids. Environ Sci Technol 44(6):2169-2175

Lowry GV, Espinasse BP, Badireddy AR, Richardson CJ, Reinsch BC, Bryant LD, Bone AJ, Deonarine A, Chae S, Therezien M (2012) Long-term transformation and fate of manufactured $\mathrm{Ag}$ nanoparticles in a simulated large scale freshwater emergent wetland. Environ Sci Technol 46(13):7027-7036

Nasseri S, Borna MO, Esrafili A, Kalantary RR, Kakavandi B, Sillanpää M, Asadi A (2018) Photocatalytic degradation of malathion using $\mathrm{Zn} 2+$-doped TiO 2 nanoparticles: statistical analysis and optimization of operating parameters. Appl Phys A 124(2): 175

Ortelli S, Costa AL, Blosi M, Brunelli A, Badetti E, Bonetto A, Hristozov D, Marcomini A (2017) Colloidal characterization of $\mathrm{CuO}$ nanoparticles in biological and environmental media. Environ Sci: Nano 4(6): 1264-1272

Pati SS, Singh LH, Guimarães EM, Mantilla J, Coaquira JAH, Oliveira AC, Sharma VK, Garg VK (2016) Magnetic chitosan-functionalized Fe3O4@Au nanoparticles: synthesis and characterization. J Alloy Compd 684:68-74

Pham TD, Bui TT, Nguyen VT, Bui TKV, Tran TT, Phan QC, Pham TD, Hoang TH (2018) Adsorption of polyelectrolyte onto nanosilica synthesized from rice husk: characteristics, mechanisms, and application for antibiotic removal. Polymers 10(2):220

Quigg A, Chin W-C, Chen C-S, Zhang S, Jiang Y, Miao A-J, Schwehr KA, Xu C, Santschi PH (2013) Direct and indirect toxic effects of engineered nanoparticles on algae: role of natural organic matter. ACS Sustain Chem Eng 1(7):686-702

Rai PK, Kumar V, Lee S, Raza N, Kim K-H, Ok YS, Tsang DCW (2018) Nanoparticle-plant interaction: implications in energy, environment, and agriculture. Environ Int 119:1-19

Ranjan S, Ramalingam C (2016) Titanium dioxide nanoparticles induce bacterial membrane rupture by reactive oxygen species generation. Environ Chem Lett 14(4):487-494

Romoser A, Ritter D, Majitha R, Meissner KE, McShane M, Sayes CM (2011) Mitigation of quantum dot cytotoxicity by microencapsulation. PLoS ONE 6(7):e22079

Rong H, Garg S, Waite TD (2019) Impact of light and suwanee river fulvic acid on $\mathrm{O} 2$ and h2o2 mediated oxidation of silver nanoparticles in simulated natural waters. Environ Sci Technol 53(12):6688-6698

Sani-Kast N, Labille J, Ollivier P, Slomberg D, Hungerbühler K, Scheringer M (2017) A network perspective reveals decreasing material diversity in studies on nanoparticle interactions with dissolved organic matter. Proc Natl Acad Sci 114(10):E1756-E1765

Selvaraj V, Bodapati S, Murray E, Rice KM, Winston N, Shokuhfar T, Zhao Y, Blough E (2014) Cytotoxicity and genotoxicity caused by yttrium oxide nanoparticles in HEK293 cells. Int J Nanomed 9:1379

Sharma VK (2009) Aggregation and toxicity of titanium dioxide nanoparticles in aquatic environment-a review. J Environ Sci Health Part A 44(14):1485-1495

Sharma VK, Zboril R (2017) Silver Nanoparticles in Natural Environment: Formation, Fate, and Toxicity. Springer, Bioactivity of Engineered Nanoparticles, pp 239-258

Sharma VK, Filip J, Zboril R, Varma RS (2015) Natural inorganic nanoparticles-formation, fate, and toxicity in the environment. Chem Soc Rev 44(23):8410-8423

Sharma VK, Sayes CM, Guo B, Pillai S, Parsons JG, Wang C, Yan B, Ma X (2019) Interactions between silver nanoparticles and other metal nanoparticles under environmentally relevant conditions: a review. Sci Total Environ 653:1042-1051

Shevlin D, O’Brien N, Cummins E (2018) Silver engineered nanoparticles in freshwater systems-Likely fate and behaviour through natural attenuation processes. Sci Total Environ 621:1033-1046

Simeone FC, Blosi M, Ortelli S, Costa AL (2019) Assessing occupational risk in designs of production processes of nano-materials. NanoImpact 14:100149

Stebounova LV, Guio E, Grassian VH (2011) Silver nanoparticles in simulated biological media: a study of aggregation, sedimentation, and dissolution. J Nanopart Res 13(1):233-244

Ubaid KA., X. Zhang, V. K. Sharma and L. Li (2019). Fate and risk of metal sulfide nanoparticles in the environment. Environmental Chem Letters: 1-15

Vishnuvarthanan M, Rajeswari N (2019) Food packaging: pectinlaponite-Ag nanoparticle bionanocomposite coated on polypropylene shows low $\mathrm{O} 2$ transmission, low Ag migration and high antimicrobial activity. Environ Chem Lett 17(1):439-445

Wan D, Sharma VK, Liu L, Zuo Y, Chen Y (2019) Mechanistic insight into the effect of metal ions on photogeneration of reactive species from dissolved organic matter. Environ Sci Technol 53(10):5778-5786

Wang K, Garg S, Waite TD (2017) Light-mediated reactive oxygen species generation and iron redox transformations in the presence of exudate from the cyanobacterium Microcystis aeruginosa. Environ Sci Technol 51(15):8384-8395

Wang P, Menzies NW, Chen H, Yang X, McGrath SP, Zhao F-J, Kopittke PM (2018) Risk of silver transfer from soil to the food chain is low after long-term (20 years) field applications of sewage sludge. Environ Sci Technol 52(8):4901-4909

Westerhoff P, Atkinson A, Fortner J, Wong MS, Zimmerman J, GardeaTorresdey J, Ranville J, Herckes P (2018) Low risk posed by engineered and incidental nanoparticles in drinking water. Nat Nanotechnol 13(8):661-669

Westesen K, Bunjes H, Koch MHJ (1997) Physicochemical characterization of lipid nanoparticles and evaluation of their drug loading capacity and sustained release potential. J Controlled Release 48(2-3):223-236

Xu J, Li J, Zhang R, He J, Chen Y, Bi N, Song Y, Wang L, Zhan Q, Abliz Z (2019) Development of a metabolic pathway-based pseudo-targeted metabolomics method using liquid chromatography coupled with mass spectrometry. Talanta 192:160-168

Yin Y, Liu J, Jiang G (2012) Sunlight-induced reduction of ionic Ag and Au to metallic nanoparticles by dissolved organic matter. ACS Nano 6(9):7910-7919 
Yin Y, Han D, Tai C, Tan Z, Zhou X, Yu S, Liu J, Jiang G (2017a) Catalytic role of iron in the formation of silver nanoparticles in photo-irradiated $\mathrm{Ag}+$-dissolved organic matter solution. Environ Pollut 225:66-73

Yin Y, Xu W, Tan Z, Li Y, Wang W, Guo X, Yu S, Liu J, Jiang G (2017b) Photo-and thermo-chemical transformation of $\mathrm{AgCl}$ and $\mathrm{Ag} 2 \mathrm{~S}$ in environmental matrices and its implication. Environ Pollut 220:955-962

Zhang D, Yan S, Song W (2014) Photochemically induced formation of reactive oxygen species (ROS) from effluent organic matter. Environ Sci Technol 48(21):12645-12653

Zhang X, Xu Z, Wimmer A, Zhang H, Wang J, Bao Q, Gu Z, Zhu M, Zeng L, Li L (2018) Mechanism for sulfidation of silver nanoparticles by copper sulfide in water under aerobic conditions. Environ Sci: Nano 5(12):2819-2829

Zhou H, Lian L, Yan S, Song W (2017) Insights into the photo-induced formation of reactive intermediates from effluent organic matter: the role of chemical constituents. Water Res 112:120-128 